\title{
$\mathfrak{Z} \mathfrak{d} \mathfrak{w} \mathfrak{d} \mathfrak{t}$

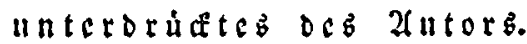

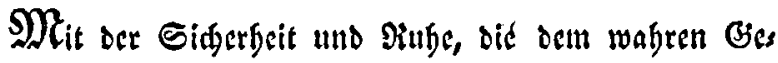
nie angeboren, úbergebe id) Der $\mathfrak{S c l t}$ meitte Biogras phie, bamit fic lerne, wie man firly zum groben sias tor bilbet, meine Portrefflidgerit im ganjen Umfange crfente, mid) licbe, fdadiçe, chre, bewuundere, und sin wenig anbete.

Golte jemand verwegen gentr finn, gegen oen getiegenen $\mathfrak{B e r t f}$ dé anßerordentlid)en Budds cinige Bweifel crleeven zul wollen, fo mag cr bedenfen, dá cr $\mathfrak{c}$ s mit cinem Siater z̆u thun bat, Der Geift, Ders ftand befight, und fajarfe Sirallen.

Borlin, im Mlai (18-).

$\mathfrak{M} \mathfrak{a} \mathfrak{r} \mathfrak{r}$.

(homme de leltres très renommé.) 


\section{- Xir -}

2. S. Das ift ju arg! - 2fudy Dns Borwort ocs 2futors, weldbes unteroricft werden follte, ift abs gecrucft! - Ė bleiot nichtz librig, ala ocn günfigen Sefer żt bitten, Daß cr bem fd)riftfellerifden Sater Den etivas foljen Ton Dicfes Borworts nid)t gu bod) anredjnen, "und bedenfen móge, Daß̧, wenn mand, wefmultbige Borrede irgend cincs andern enpfind amen 2futors ill Dic wabre Eprache oct intigen Şarzettbs meinumg ḣberfegt werben folle, sả nicht viel anders Gransfommen würe.

D. \$ุ. 